\title{
A Computational and Experimental Study of Fluid Flow in a De-duster
}

\author{
QIANPU WANG, MORTEN CHR. MELAAEN and \\ SUNIL R. DE SILVA $\dagger$
}

Keywords: CFD, air classificr, de-dusting, de-dluster

A three-dimensional computational fluid dynamics model with an RNG $k$-i; model and second upwind scheme in the Fluent package was used to simulate the flowpattern in a de-duster. The model predictions for the flow field inside the dispersion device were comparcd with LDA measurements. The primary objectives are to understand such complex flow ficlds and to guide the development and optimisation of this de-duster.

From the simulation results. the old prototype appears to have some disadvantages and shortcomings. A modification is proposed here to overcome some of these. The major new development in modification is the introduction of a second air inlet and the optimisation of the de-duster geometry. The modification will minimize the disadvantages and shortcomings identified so far.

\section{Introduction}

De-dusting is the separation of very fine particles (generally under $100 \mu \mathrm{m}$ ) from a mass of larger particles or granules. In other words a form of classification. The background for the development of a de-duster, was that the Department of Powder Science and Technology Research (POSTEC), at the Telemark Technological R\&D Centre (Tel-Tek) had developed an enhanced dispersion device for use with air classifiers which later proved to be more suitable for de-dusting than for dispersion. The unit is shown in Figure 1.

The first de-dusting trials with the unit were undertaken with polythene pellets of about $4 \mathrm{~mm}$ in diameter being cleaned of dust and angel hair. The results were promising, with considerable dust reduction. even at very high feed rates. The unit was then used to separate fine ash-particles from sand-particles. It worked quite well at moderate feed rates. but there was a considerable carry over of coarse particles into the fine fraction. It was also used in tests to remove particles from the surlace of fish pellets bonded by oil. In this calse, however, the unit damaged the pellets. Finally, the unit was used to de-dust a batch of explosives. The specification called for less than $0.1 \%$ of dust, whereas the material sent to a customer had $0.3 \%$. After the out-of-specification material was cleaned, the dust content was far below the limit specified by the customer. The major challenge in improving the unit appears to be to prevnt fine particles leaving with the coarse fraction, and the coarse particles leaving with the fine fraction. In order to do this, and for purposes of scale-up. at better understanding of the nature of the flow in the unit is required.

The primary objectives of this work, therefore, were to understand the complex

* An carly version of this article wals presented at the SIMS 2001 Conlerence. October 8th-9th 2001, Porsgrunn. Norwaly.

†Department of Technology. Telemark University College/Tel-Tek, Kjolnes Ring. N-3914 Porsgrunn. Norway. 


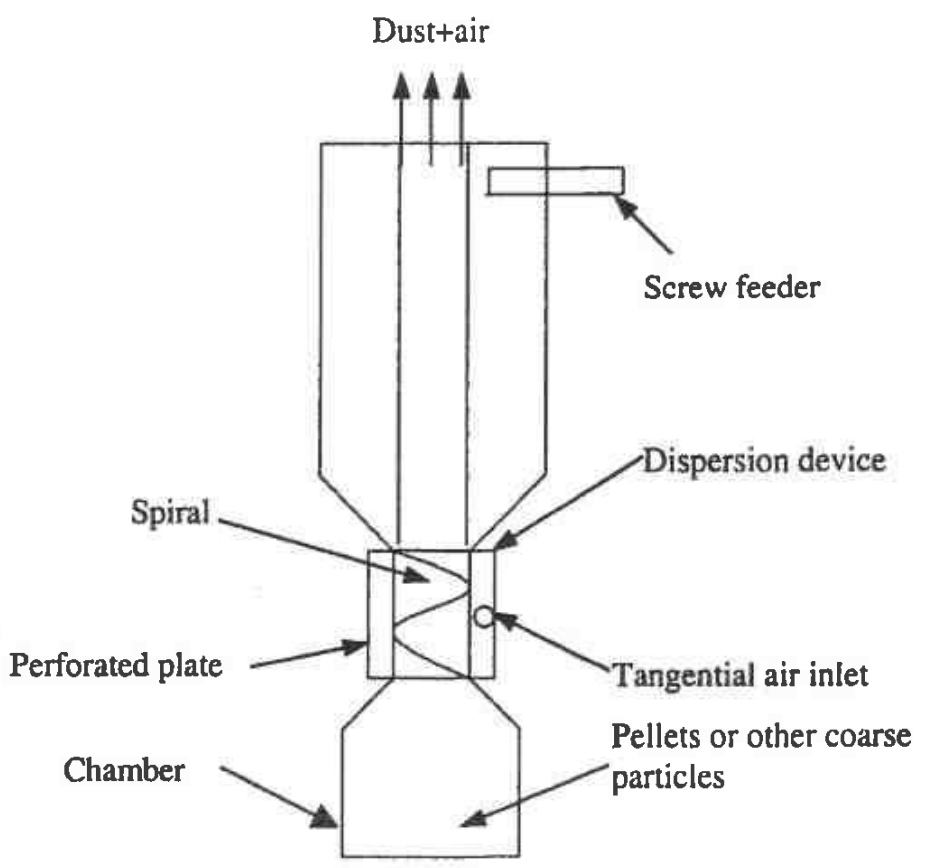

Figure 1. Schematic representation of the de-duster.

flow fields prevailing in the unit, and to provide complete benchmark data for comparisons with numerical predictions based on practical models for turbulent swirling flows, and thereby permitting the optimisation of the geometry of the deduster and providing guidelines for the further development and scale up of the unit. Here only the de-dusting of materials on which fine dust adheres strongly to the larger particles is considered.

Flow fields of the de-duster under various set-up conditions were measured by using laser Doppler anemometry (LDA). Using the Fluent package, a three-dimensional computational fluid dynamics model has also been developed. Discussions and analyses of the experimental, as well as the computational results, are presented.

\section{Principle of separation}

The unit that was used is shown in Figure 1. The dispersion zone consists of a cylindrical section with perforated walls into which is mounted at spiral. The pellets which fall down in the main separator drop onto the surface of the spiral and move along its surface until they reach the exit. During their passage, they are exposed to high velocity jets of air which are produced by the specially perforated wall of the cylinder. The holes in the wall are constructed in such a way as to induce a very strong vortex that induces the pellets to move down the spiral rather than simply falling out of the separator. Thus the spiral increases the residence time of the pellets in the vortex, enhancing dust removal.

The perforated wall used in the enhanced dispersion device was a Conidur plate, a type of perforated sheet that is being increasingly used in screening processes. The main difference between Conidur and conventional perforated plate is that the holes are triangular to semi-elliptical with conical, slanting openings in the direction of flow, rather than round. These holes create a raised, rough surface. 
This allows small holes to be made in relatively thick sheets of steel or aluminium. Smaller holes give the larger velocity required by the process. The conical apertures also reduce flow resistance due to clogging, and have an increased screening capacity. There are two types of holes: fine-holed, that have triangular to semi-elliptical perforations, and slotted holes that have slit openings that give larger open-screen surfaces. The raised surface can be reduced with light or heavy rolling, and polishing can reduce roughness. The plates are manufactured by the German company Hein. Lehman of Dusseldorf.

The performance of the de-duster depends strongly on the flow field in the dispersion device, especially the tangential velocity distribution. The classical theory proposed by Rumpf (1975) states that particle separation occurs when the adhesion forces are supplanted by the applied removal force, causing a simultaneous breakage of interparticle links along the rupture plane. The determination of the adhesive forces between particles is of great interest to a wide range of industrial operations. But it is very difficult to determine the adhesive forces between particles, especially for different size particles. Hamaker (1937) carried out calculations for various geometries. The adhesive force between two spheres is expressed by

$$
F=\frac{A d_{p}}{12 z^{2}}
$$

where

$$
d_{p}=\frac{d_{1} d_{2}}{d_{1}+d_{2}}
$$

and where $z$ is the separation distance at the point of contact, $d_{1}$ and $d_{2}$ are the diameters of the two particles. $A$ is referred to as the Hamaker constant. Czarnecki and Dabros (1980) suggest that the effect of roughness of the sphere surfaces modifics the van der Waals force by

$$
F=\frac{A d_{\bar{p}}}{12(z+b)^{2}}
$$

where $b=\left(b_{1}+b_{2}\right) / 2$ and $b_{1}$ and $b_{2}$ are the heights of irregularities on the two spheres.

Here a calculated result given by Rumpf (1975) is used to estimate the particle particle adhesive force. For the same size particles, conventional material parameters give the relationship between adhesive force and particle size that is shown in Figure 2. It shows that for fine particles with size less than $100 \mu \mathrm{m}$. the weight force of at particle is several orders of magnitudes smaller than the universal adhesive forces, so

Here a calculated result given by Rumpf (1975) is used to estimate the particle

We also know, from experience, the phenomenon of electrostatic charge, which often leads to undesirable adhesive forces especially in non-conductive materials. When particles of different materials are brought into contact with one another: electrons pass from one material into the other. Because the partners become oppositely charged. they also attract one another. In the case of very fine particles. electrostatic forces are an order of magnitude smaller than the van der Waals forces. and are therefore generally negligible.

The removal forces in the de-duster for the dust are drag force. accelerationdependent drag terms (the added mass and Basset history integral terms) and the lift forces (such as the Magnus and Saffman terms) etc., amongst which the drag force dominates. 


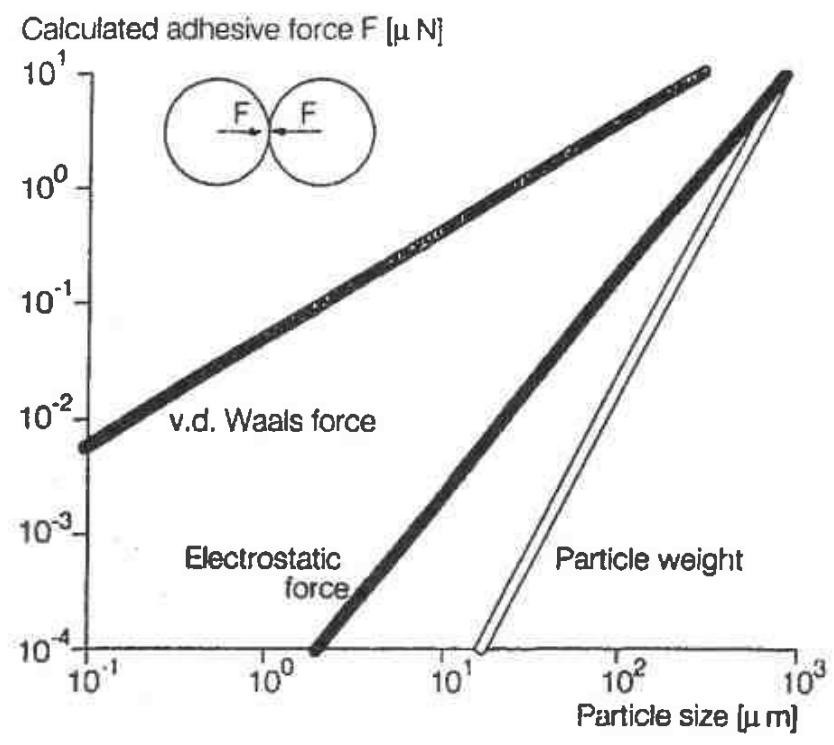

Figure 2. Adhesive forces and particle weight force given by Rumpf (1975).

$$
F_{D}=C_{D} A \frac{\rho_{g}}{2}\left|\vec{u}_{g}-\vec{u}_{p}\right|\left(\vec{u}_{g}-\vec{u}_{p}\right)
$$

where $A$ is the exposed frontal area of the particle to the direction of the incoming flow and $C_{D}$ is the drag coefficient, which is a function of the particle Reynolds number. Here the drag coefficients for particles are based on the work by Wen and Yu (1966)

$$
C_{D}=\frac{24}{\operatorname{Re}_{s}}\left[1+0.15 \operatorname{Re}_{s}^{0.687}\right]
$$

If $F_{D}$ is equal or larger than adhesive forces, then the separation will happen. From Figure 2, for fine particles with diameter about $50 \mu \mathrm{m}$, the adhesive force between particles is about $10^{3}$ times larger than the particle weight. To separate the two particles

$$
F_{D}=C_{D} A_{2}^{\rho_{g}}\left|\vec{u}_{q}-\vec{u}_{p}\right|\left(\vec{u}_{! j}-\vec{u}_{p}\right) \geqslant 1000 F_{a}
$$

where $F_{q 1}$ is the weight force of the particles.

To separate fine particles with diameter $20-50 \mu \mathrm{m}$, the velocity difference should be between $10-18 \mathrm{~m} / \mathrm{s}$. This means the flow field inside the dispersion device is critical to the separation.

The experiments with LDA were carried out with the laser having a focal length of $400 \mathrm{~mm}$. The measured control volume is $0.18 \times 0.18 \times 3.8 \mathrm{~mm}$, small enough to get good results. Very fine droplets (dry ice) are used as tracers. The size of these droplets is about $1-2 \mu \mathrm{m}$. The velocities at 8 cross sections with 20 points in each section in different positions inside the dispersion device shown in Figure 3 were measured.

\section{Numerical approach}

The problems in simulating a strongly swirling, confined and turbulent flow numerically are well known. The widely used $k-k$ model does not yield realistic 


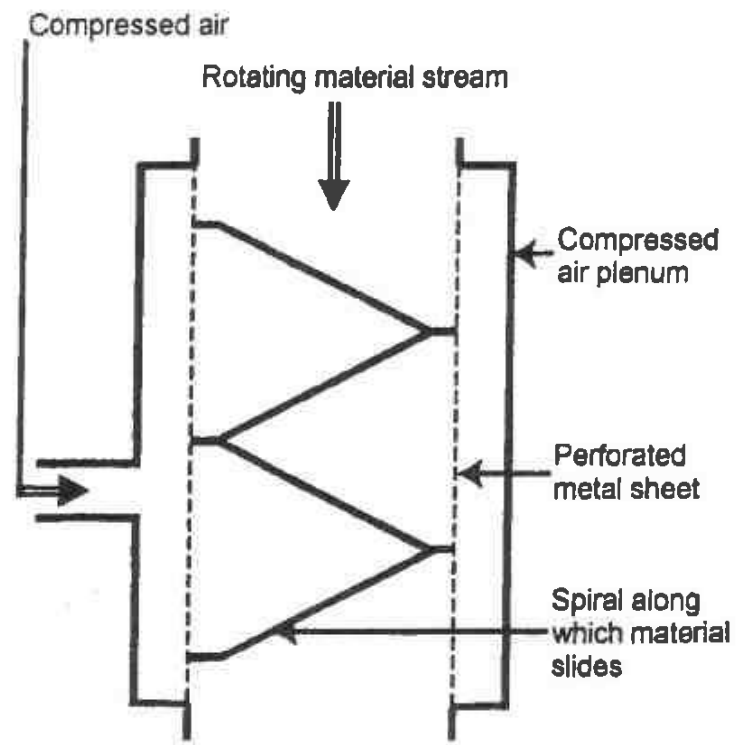

Figure 3. Dispersion device developed by POSTEC.

velocity profits. When choosing a simulation technique, three considerations have to be made:

- Dimensionality of the simulation (2-D axisymmetric or 3-D)

- Method of turbulence modelling

- Method of discretization.

The de-duster is one of the unit opcrations which lends itself most naturally to 2-D axisymmetric CFD simulations if without spiral. Here a 3-D simulation is used because of the spiral. As regards the discretisation schemes it is often referred to the order of accuracy, and the power law scheme is a first order scheme and the Quick scheme (Fluent, 1996) is a higher order scheme. The decision of what discretization scheme to use, is somewhat double-edged. When a higher order scheme is used in which the error is smaller than a first order scheme, the residuals may become unstable, and a converged solution hard to achieve. When a first order scheme is used, the solution process may be casier, but the numerical error may be significant. Unfortunately it was impossible to obtain convergence by using Quick in this calse. So the second order upwind scheme is used.

Turbulence modelling is also an important matter in the simulation of the deduster. The most popular turbulence model in CFD modelling has been the standard $k-\varepsilon$ model. This model has, however, been shown to be inappropriate for simulation of strong swirl flow. The standard $k-8$, model leads to overprediction of the turbulent viscosity and a major deviation in the velocities occurs. The RNG $k-8$ model is at turbulence model which is based on statistical mechanics rather than continuum mechanics, and offers modifications that make this model useful in swirl flow. The RNG $k$-r: model has similar equations for the $k$ and the ( equations as the standard $k-i$ model, but with dillerent values for the constants. Additional terms exist in the b-equation. For simulations of strong swirl flow, modifications to the calculation of the effective viscosity are most significant. The effective viscosity is ealculated from an ordinary differential equation which includes the eflect of swirl. It is expected that the RNG $k$-6: model still will have some deviations in the aceuracy, since anisotropic 


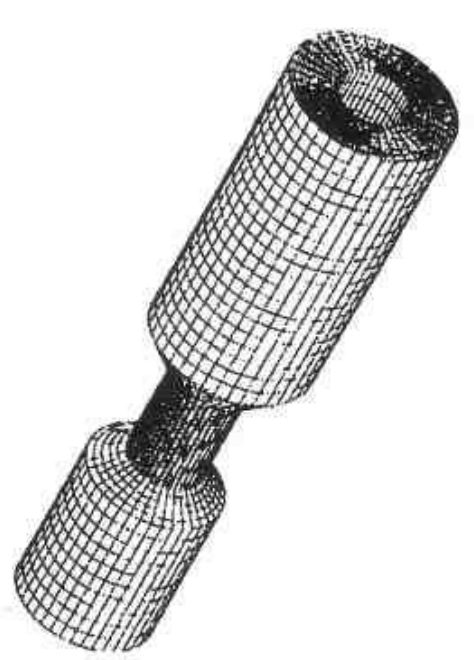

Figure 4. Calculation domain with grid nodes.

effects are still not included in the description of the turbulence. In order to consider the anisotropic description of the turbulence, the Reynolds Stress Model (RSM) is a natural choice. This model solves the individual Reynolds stresses with a differential equation for each of the stresses. This leads to 7 differential equations, 6 equations for the Reynolds stresses and one for the dissipation, and this makes the RSM model computationally expensive. It is also very hard to get converging results. The RSM model was ever tested first. Unfortunately it is impossible to get a convergence. Therefore the RNG $k-\varepsilon$ model is used here. The calculation domain with grid nodes are shown in Figure 4.

In the simulation of the de-duster, the major problem is how to deal with the perforated plate which is set as velocity inlet because of the special shape and very small size $(0.1-1 \mathrm{~mm})$ of the perforations. It is impossible to produce a so fine grid in a 3-D problem because of computer capacity right now. It is also unreasonable to use a uniform velocity. The inlet grid nodes are shown in Figure 5. The commercial computer code Fluent 4.5 is used in the computations, and the numerical methods are described in detail in Patankar's book (1980). The model and discretisation schemes are given in detail in the Fluent user guide (1996).

The total inlet area in the simulation is 10 times larger than in the actual perforates

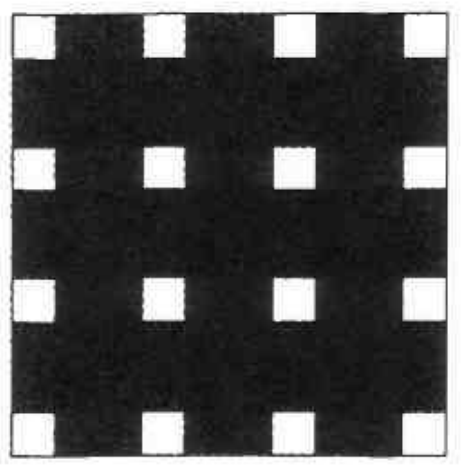

Figure 5. Inlet grid area. 
platc. The tangential velocity is calculated by the volume flow rate and the total actual open area in the perforated plate. The radial velocity is calculated by the volume flow rate and the total inlet area in the simulation to keep the volume flow rate the same as the measurement. The tangential inlet velocity is $35 \mathrm{~m} / \mathrm{s}$ and the radial velocity is $0.85 \mathrm{~m} / \mathrm{s}$. At the outlet, the static pressure is set to zero.

Inlet turbulence is set via input of turbulence intensity and characteristic length. The turbulence intensity is defined as the ratio of the root-mean-square turbulence velocity fluctuations to the mean flow velocity $\left(u^{\prime} / u_{u r g}\right)$, generally expressed as a percentage which is set as $2 \%$ here. The inlet value of $k$ is calculated from the specified inlet turbulence intensity, $I$ as follows:

$$
k=\frac{3}{2}\left(u^{\prime}\right)^{2} \quad \text { or } \quad k=\frac{3}{2}\left(u_{\text {arg }} I\right)^{2}
$$

where $u_{\text {arg }}$ is the magnitude of the mean velocity specified at the inlet. Then $\varepsilon$ is calculated based on the turbulence length scale, $l$ as follows:

$$
\varepsilon=C_{\mu}^{3 / 4} \frac{k^{3 / 2}}{l}
$$

where $C_{\mu}$ is an empirical constant (approximately 0.09 ). The turbulence scale $l$ is decided by the physical size. A typical relationship between $/$ and the physical size $L$ is

$$
l=0.07 L
$$

where $L$ is the size of inlet grid line shown in Figure 5.

\section{Results and discussion}

First the simulation results which use the RNG $k-\varepsilon$ model, the second order upwind scheme and specified inlet boundary condition are compared with the measurement results inside the dispersion device to verify the reliability of the model and specified boundary conditions. Then flow simulations are used to find the weakness of the first prototype. A new optimised de-duster is suggested.

\subsection{Comparison between simulation and measurement}

Measured mean air tangential velocity profiles are shown in Figure 6. Figure 7 gives a comparison of tangential velocity distributions obtained by measurements

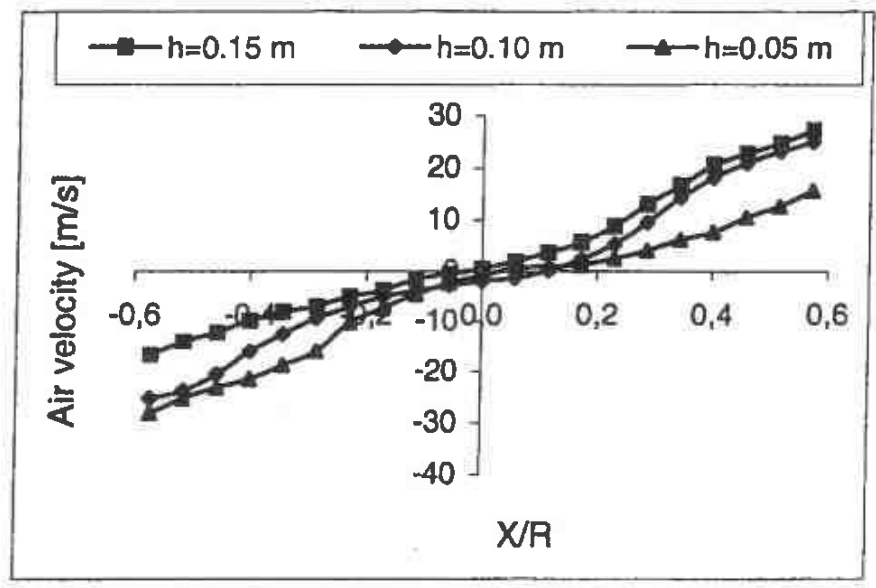

Figure 6. Mean measured air tangential velocity profiles at different heights inside dispersion device. 

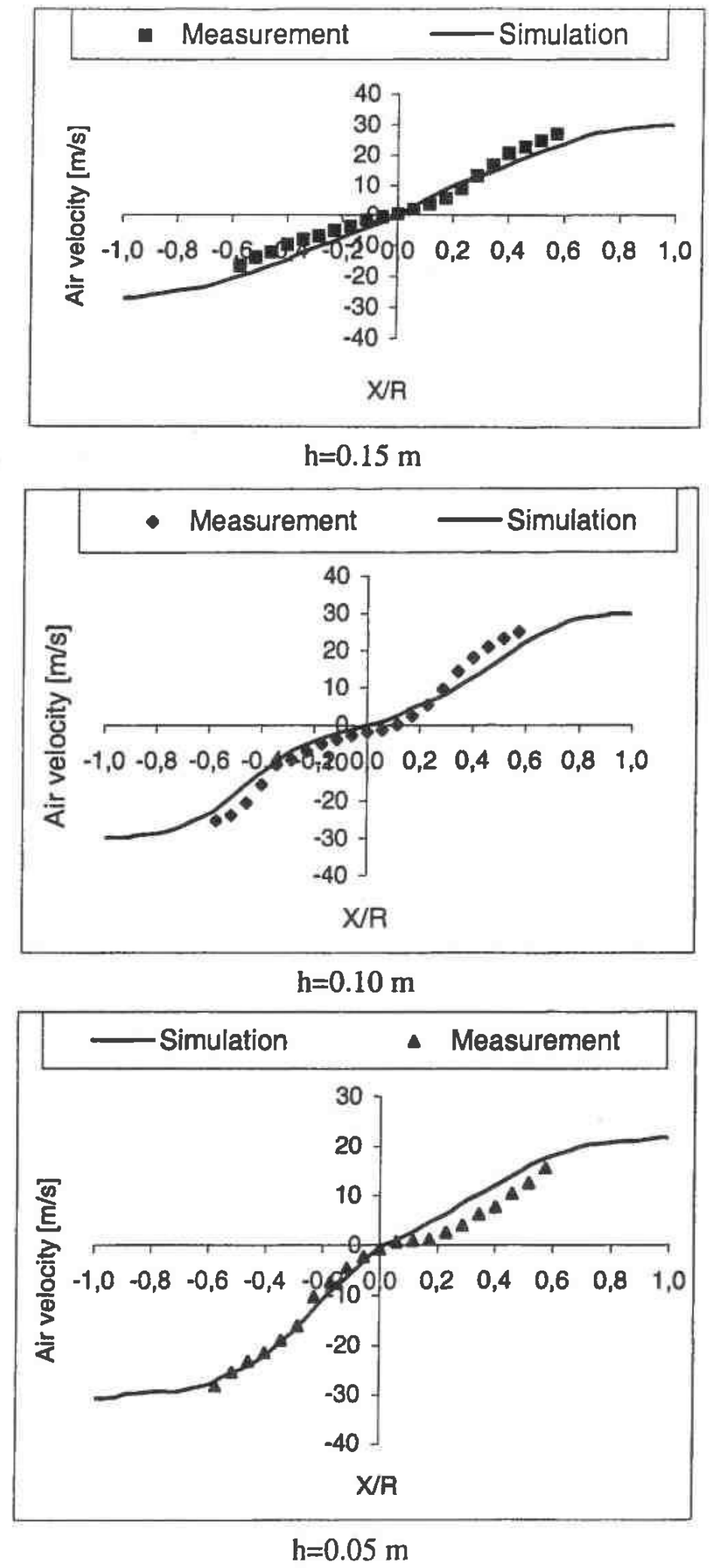

Figure 7. Comparison of tangential velocity distributions between simulation and measurement at three heights above bottom of the dispersion device. 
and numerical predictions. at three different positions inside the dispersion device. $h$ is the height from the bottom of the dispersion device to the measured section.

The results show that LDA measurements and numerical predictions using the RNG $k-\varepsilon$ model and the second order upwind scheme are in reasonable agreement. Some errors are expected because of the simplification of the inlet boundary condition.

\subsection{Optimisation of the de-duster}

First the effect of spiral on the flowpattern is tested. As mentioned above, the purpose of the spiral is expected to increase the residence time of the pellets or other particles in the vortex, and hence enhance dust removal. Simulations of the flowpattern in the de-duster with and without spiral are shown in Figures 8,9 and 10, respectively. These figures show vector plots of the axial and tangential velocity components predicted under the same conditions.

The main difference between the two simulations is that the velocity distribution with the spiral is not axisymmtric. Figure 10 shows that the tangential velocity decreases from the perforated wall to the centre inside the dispersion device. The dedusting, however, is meant to occur at the wall.

Figures 8 and 9 show an interesting result, in that the axial velocity close to the perforated wall is downward. The line particles or dust will travel down with the coarse particles and be collected in the chamber if they are not separated along the length of the dispersion device. Some of the line particles with a size less than $10 \mu \mathrm{m}$ will probably go up again with the main stream at the centre. Although the residence time of particles is increased by the spiral. The effective residence time of particles is very short no matter whether or not a spiral is used, due to this downward flow. To enhance the performance of the de-duster, therefore, it is necessary to ensure that the fines are separated and carried out of the dispersion zone before reaching the coarse collector.
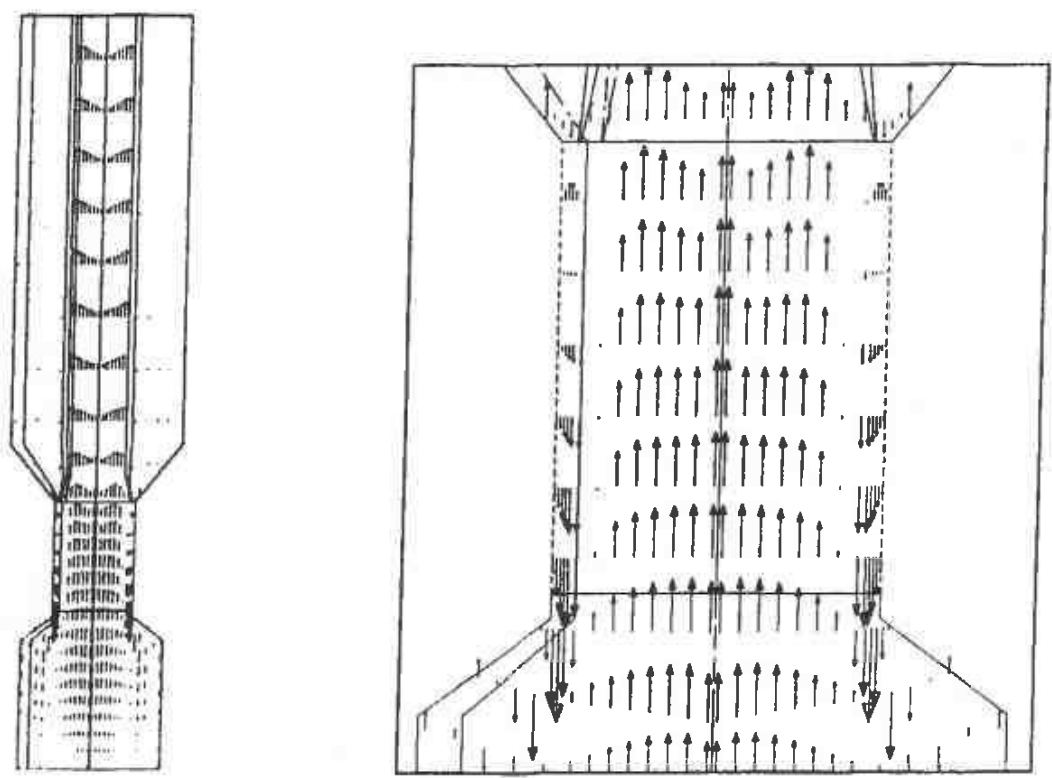

Figure 8. Axial velocity distribution (without spiral). 

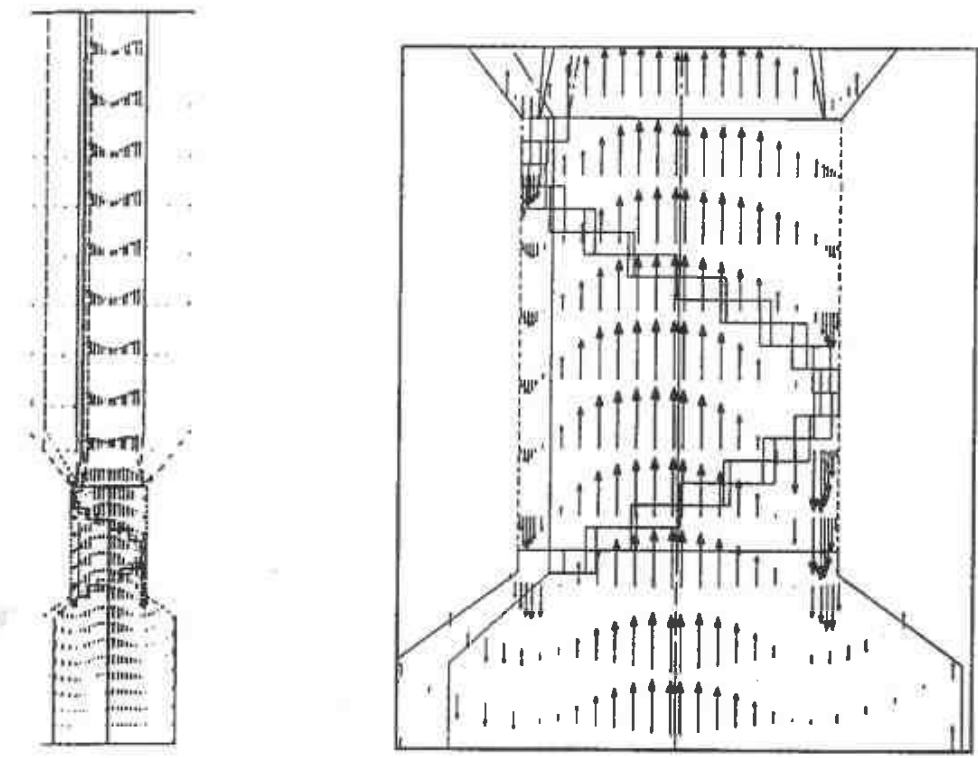

Figure 9. Axial velocity distribution (with spiral).

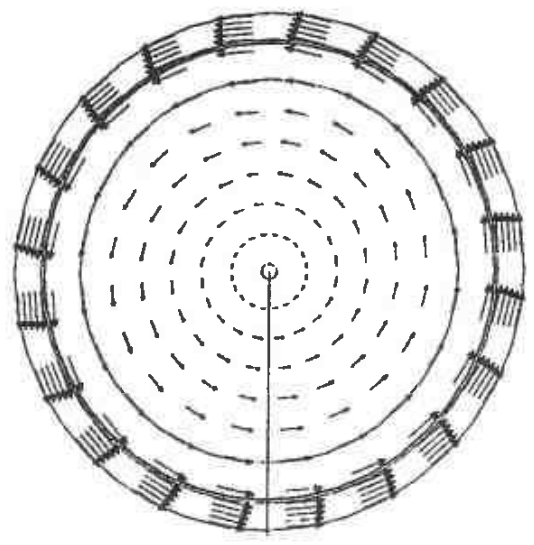

without spiral

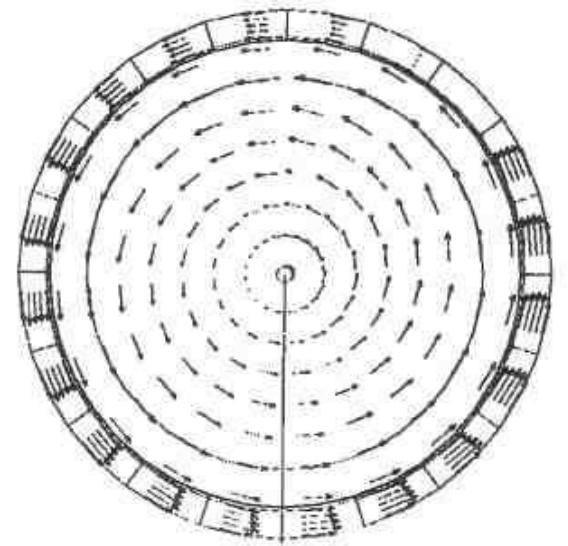

with spiral

Figure 10. Comparison of tangential velocity distributions without and with spiral.

A modification which would ensure this is shown in Figure 11. The dispersion device is as it was. The modification is that an additional air flow is injected from the bottom of the de-duster. The particles are fed from the top of the de-duster. The effect of this modification is shown in Figure 12 which reveals that the downward axial velocity close to the perforated wall has been be eliminated, hence increasing residence time. The proposed tangential entry of the feed will ensure that the particles will move close to the wall before entering the dispersion device due to the strong swirl flow in the upper part of the de-duster. Both the upper part of the de-duster and the dispersion device are the effective area for de-dusting. The inlet particle velocity and direction will not be sensitive to the de-dusting efficiency any more. This modification is particularly suitable for the separation of particles which the adhesive forces between the fine and coarse particles arc particularly strong. 


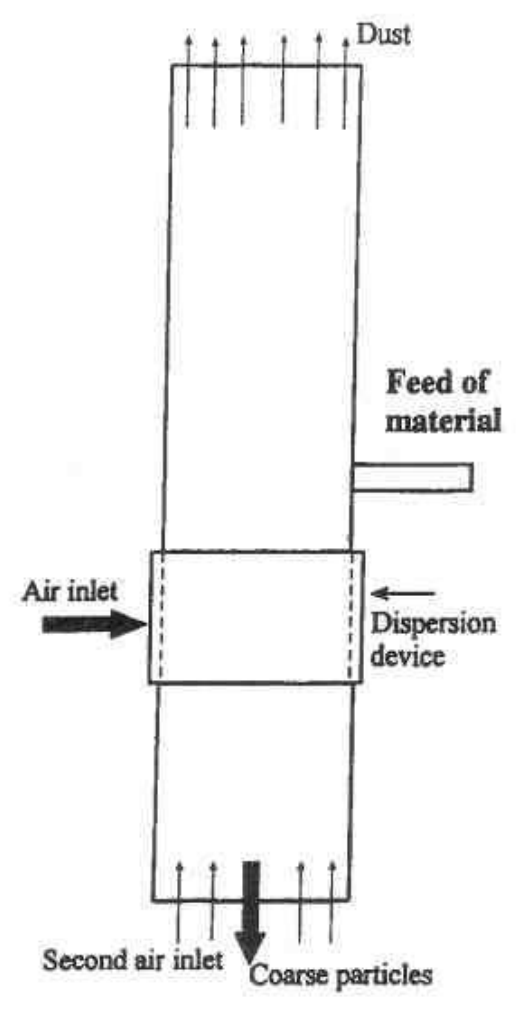

Figure 11. Schematic representation of the new modified de-duster.
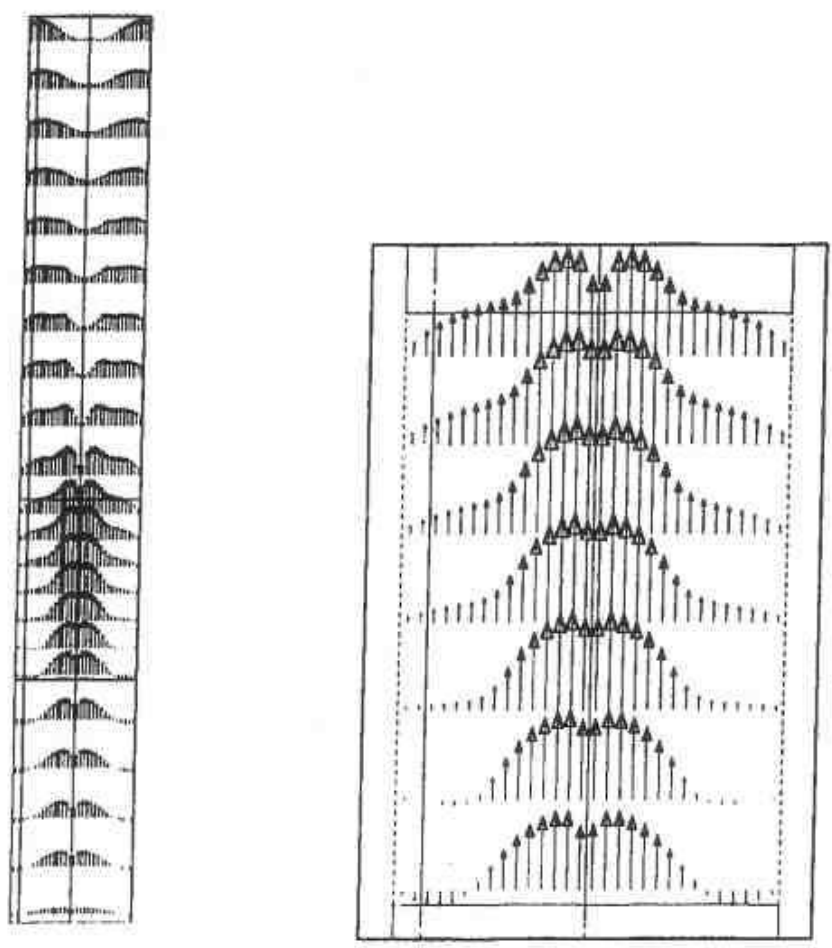

Figure 12. Axial vclocity distribution in the new de-duster. 


\section{Conclusions}

A three-dimensional computational fluid dynamics model with the RNG $k-8$ model and the second upwind scheme in the Fluent package were used to simulate the air flowpattern in a de-duster. The model predictions for the flow field inside the dispersion device were compared with LDA measurements and a reasonable agreement was observed when using specified inlet boundary conditions.

From the simulation results, the current prototype has some disadvantages and shortcomings. A modification is proposed here to eliminate the most serious of these. The major new development is the introduction of a secondary air flow at the bottom of the unit and the optimisation of the de-duster geometry. The modification will minimize the disadvantages and shortcomings identified so far. Of course the new de-duster needs further testing by measurement.

\section{REFERENCES}

CZARneCKi, J. and Dabros, T. (1980). Attenuation of the van der Waals attraction energy in the particle/semi-infinite medium systems due to the roughness of the particle surface. J. Colloid Interface, 78, 25.

Fluent USER's GUIDE (1996). Fluent Inc., Lebanon, New York USA, 4.4 edition.

HAMAKER, H. C., (1937). The London-Van der Waals' attraction between spheriodn particles. Physica, 4, 1058.

Patanker, S. V., (1980). Numerical heat transfer and fluid flow. Taylor \& Francis.

RumpF, H. (1975). Mechanische Verfahrenstechnik. Carl Hanser Verlag, Munchen-Wien.

Wen C. Y. and Yu Y. H. (1966). Mechanics of Fluidization. Chem. Eng. Prog. Symp. Series, $62,100-111$. 